\title{
Streptococcal infection complications in laryngology and dentistry-review
}

\author{
Michał Michalik ${ }^{1}$, Agnieszka Laskus ${ }^{2 *}$ and Adrianna Podbielska-Kubera ${ }^{1}$ \\ ${ }^{1}$ MML Medical Center, Bagno street 2, 00-112 Warsaw, Poland \\ ${ }^{2}$ Trio-Dent Dental Clinic Polna street 3, 00-622 Warsaw, Poland
}

\begin{abstract}
Oral and pharyngeal biofilm formed by oral bacterial flora, is comprised of over 700 microbial species forming a human-oral microbiome. Most oral microorganisms are non-pathogenic opportunistic commensals to maintain oral health condition.

Streptococci, the first microorganisms colonizing oral surfaces and dominant in the human mouth, are the physiological flora of the oral cavity. However, they may spread to other areas of the facial skeleton and cause serious complications inter alia: otitis media, sinusitis, encephalitis, cerebral abscesses, purulent craniofacial infections Streptococci, as a component of the biofilm in dental plaque, are often responsible for dental problems such as: caries, pulpitis, endodontic problems, periapical lesions around teeth roots, periimplantitis and periodontal diseases involving tissues supporting the teeth: bone, ligaments, root cementum, gingivae. There is a strong relationship between caries, root canals biofilm, periodontal diseases and facial skeleton complications, but also a significant impact of dental and periodontal problems on the overall health of patients. Pathogenic factors from oral Streptococci such as extracellular polymeric substances, proteins, nucleic acids and toxins affect the development of inflammation and consequently, various systemic diseases, such as infective endocarditis, atherosclerosis, cardiovascular diseases, diabetes, intestinal inflammation, aspiration pneumonia, autoimmune diseases, as well as bacteraemia.
\end{abstract}

However, the pathogenicity of Streptococci is still not fully understood and is the subject of further studies. An important problem is the increasing resistance of streptococci to antibiotics as well as their ability to produce virulence factors. They are often treated as commensal bacteria, which delays and worsens further diagnostics and contributes to serious complications.

Literature data indicate that Staphylococci and Streptococci are the most common microorganisms in intracranial complications. The presence of anaerobic bacteria or multi-microbial infections within sinuses are the factors determining the more severe course of the infection, most often with the necessity of undergoing a surgical procedure, extended hospitalization, and application of intravenous antibiotic therapy.

\section{Introduction}

The creation, development and role of bacterial biofilm in the etiopathogenesis of laryngological diseases and diseases of the oral cavity has been and still is the subject of numerous research works, and the fact of the complex structure and adaptation mechanism of bacteria raises a number of questions concerning the effectiveness of treatment of the diseases caused by them.

The oral cavity health of an individual depends on the presents of healthy oral biofilm of native microorganisms on surface of teeth, gingiva, buccal mucosa and dorsum of the tongue. Dental plaque on tooth surface and oral mucosa is characterized by dynamic metabolically structure and is microbial communities embedded in a self-produced matrix of extracellular polymeric substances.

In dentistry, the disruption of this microbial homeostasis, as well as unbalanced microflora, excessive growth or increase in the number of acidogenic bacteria in correlation with changes in localoral environment ( type of diet, hygienization, saliva viscosity, salivary flow) and host immune response cause the development of the most common diseases of the oral cavity, i.e. dental caries, dental pulp diseases, periodontal diseases and peri-implant infective problems.

The presence of bacterial biofilm on the walls of the root canal or at the root apex, on its external wall, causes periapical complications (apical periodontitis) as well as may cause endodontic treatment failures. Endodontal and periodontal infections are always associated with complex microfloras for example in apical periodontitis 200 species have been encountered but in marginal periodontitis up to 500 species that are most often gram-negative anaerobic but from grampositive Sreptococcus intermedius, S.sanguis, S.constellatus were isolated. In development of dental caries S.mutans, S.mitis, S.salivarius are strongly involved.

All this oral bacterial infectious diseases are odontogenic factors of further craniofacial- area complications, for example complications within the maxillary sinuses. In addition they significantly affect, using direct and indirect-two major pathways, various systemic diseases therefore reliable understanding the mechanisms of this strong dependence would lead to the development of novel preventive measures that are so important to patient's health. In laryngology, bacterial inflammation of the nasal and sinus mucosa is most often a consequence of viral infections of the upper airways. It may also occur after odontogenic infections, surgeries, or injuries [1]. The symptoms

${ }^{*}$ Correspondence to: Agnieszka Laskus, Trio-Dent Dental Clinic Polna street 3, 00-622 Warsaw, Poland, E-mail: agnieszka@laskus.pl

Key words: periodontal diseases, streptococci, facial skeleton complications, dental caries, chronic sinusitis, periimplantitis, dental plaque, biofilm

Received: June 29, 2020; Accepted: July 13, 2020; Published: July 16, 2020 
of viral inflammation of the nasal and sinus mucosa usually disappear spontaneously. The transition of the disease to bacterial sinusitis usually requires a specific antimicrobial therapy [2].

Chronic sinusitis (CS) is a multifactorial disease that affects people of all ages. The diagnosis of CS is based on subjective symptoms, their duration and objective evidence of inflammation. The main symptoms of CS include: congestion of the nasal mucosa, abundant secretion, weakened sense of smell, discomfort [3]. The heterogeneous nature of this disease, influenced by genetic factors, immune response of the host and composition of sinus microbiome, makes it difficult for researchers to explain the pathophysiology of CS [4]. Chronic right maxillary sinusitis is illustrated in Figures 1-6.

For a long time, the sinuses of a healthy person were believed to be a sterile environment. However, numerous studies have shown that not only the sinuses of patients with CS, but also healthy control patients show the presence of bacterial colonies [5]. The physiological bacterial flora of the sinuses includes strains of: Streptococcus pneumoniae,

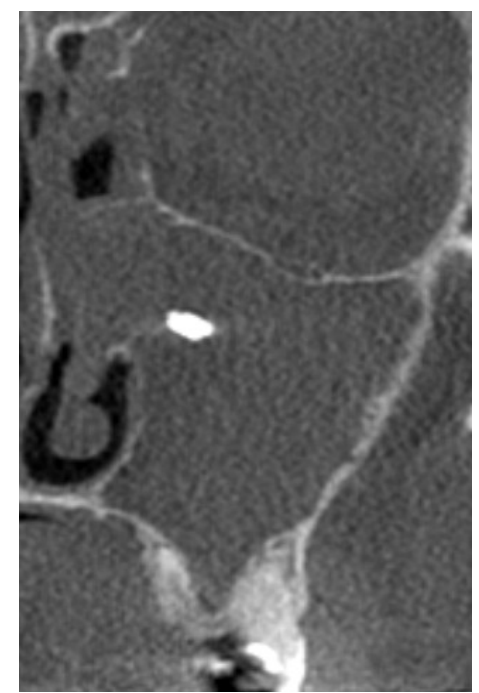

Figure 1. Foreign body in the left maxillary sinus with massive inflammation present

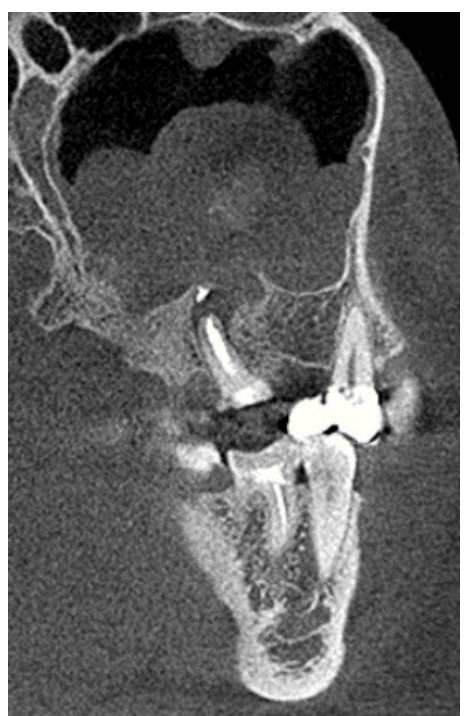

Figure 2. Polypoid sinusitis with the presence of a foreign body and cyst at the root of the tooth treated endodontically

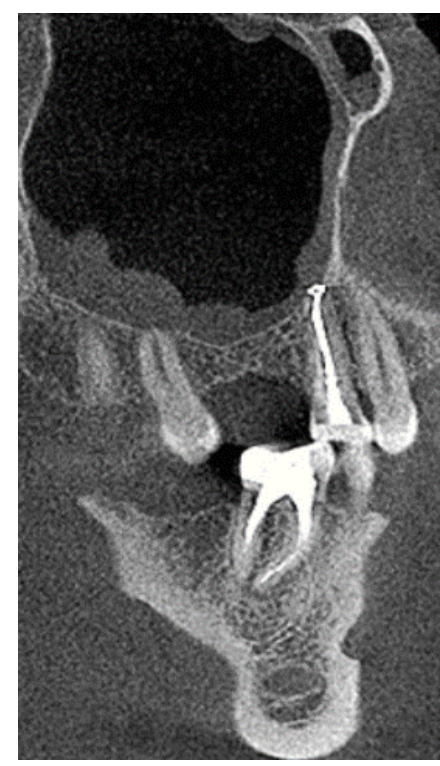

Figure 3. Reactive sinus mucositis after endodontic treatment of premolar

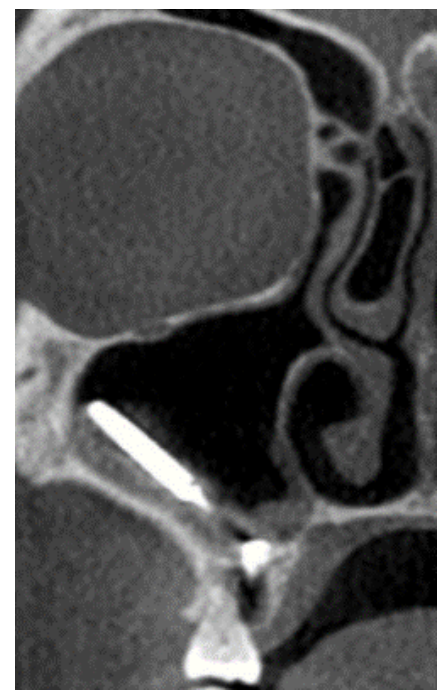

Figure 4. Right maxillary sinus - foreign body with inflammation

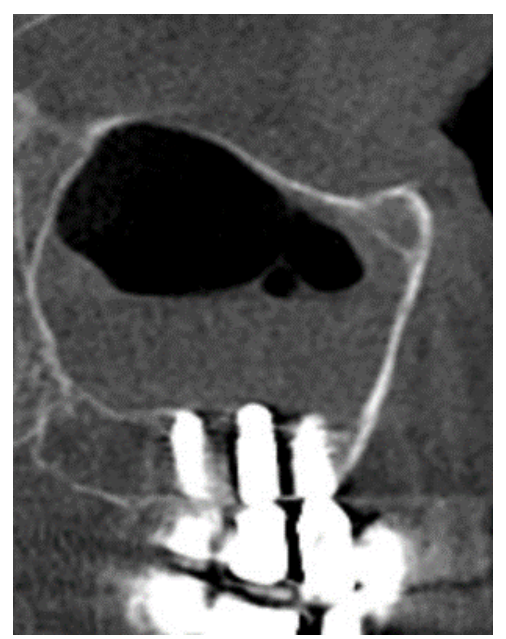

Figure 5. Reactive sinus mucositis after implantological treatment 


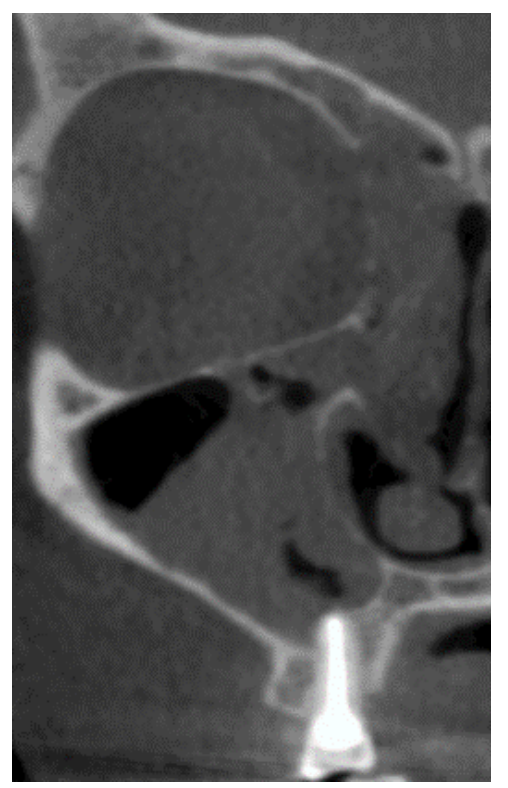

Figure 6. Chronic sinusitis of the right maxillary sinus

Haemophilus influenzae, Staphylococcus aureus, coagulase-negative staphylococci, and anaerobic bacteria such as Bacteroides, anaerobic Gram-positive cocci. The presence of these bacteria is "normal" if their quantities are low. When the bacterial index (BI) exceeds 1000 colony forming units per milliliter of mucus $(\mathrm{CFU} / \mathrm{ml})$, they are considered pathogenic [6].

In the case of acute sinusitis, one species of usually aerobic bacteria dominates the sinuses. In the case of CS, single microorganism infections are rare. Mixed bacterial flora with the presence of 2-3 bacterial strains is most common [5].

The main pathogens in acute bacterial sinusitis are S. pneumoniae, $H$. influenzae and M. catarrhalis. Additional pathogens include other streptococcus species, S. aureus and anaerobic bacteria. Unlike other sinuses, acute sphenoid sinusitis is usually characterized by the presence of S. aureus strains [7]. Gram-negative bacteria such as: Pseudomonas aeruginosa, Proteus sp., Klebsiella sp., Enterobacter sp., Escherichia coli are less common in CS [6].

The most common bacteria causing pharyngitis and tonsillitis are Streptococcus sp., Corynebacterium diphtheriae, Neisseria gonorrhoeae, Chlamydia pneumoniae and Mycoplasma pneumoniae [8].

\section{Characteristics of Streptococci}

Bacteria of the Streptococcus (streptococci) genus include microorganisms that inhabit the mucous membrane of humans and animals. Streptococci are Gram-positive bacteria, cocci that form characteristic pairs and chains. These bacteria constitute the physiological flora of the oral cavity and intestines in humans and animals [9]. They also can be found on the skin and in the upper airways. They can spread from the throat to the surrounding tissues, leading to purulent complications such as otitis media, mastoiditis and tonsillitis. In rare cases, they may also lead to meningitis [10].

Streptococcus infections are particularly important to public health [11]. Streptococci are identified as etiological factors of serious diseases: arthritis, pneumonia, peritonitis, endocarditis, sepsis, as well as social diseases of the oral cavity such as dental caries or periodontal diseases [10].
Streptococci are divided into 49 species and 8 subspecies. As many as 35 of them are sources of invasive infections in humans [9]. Microbiologists grouped the bacteria by analysing the growth of their colonies on blood agar plates. Streptococci form three characteristic hemolysis patterns: alpha (partial), beta (complete) and gamma (no hemolysis).

Alpha-hemolysis is caused by the iron reduction in hemoglobin, resulting in a green colour in the bloody agar plate. Alpha-hemolytic streptococci are mainly S.pneumoniae and viridans streptococci. (Latin name: Streptococcus viridans). Viridans streptococci may also belong to $\gamma$-hemolytic bacteria (i.e. non-hemolytic bacteria). The viridans streptococcal species include: Streptococcus mitis, Streptococcus oralis, Streptococcus sanguinis, Streptococcus salivarius, Streptococcus mutans. They are part of the physiological bacterial flora of the human oral cavity.

Beta-hemolysis is associated with complete rupturing of erythrocytes, which results in a distinctive bright area around the bacterial colonies. Beta-hemolytic streptococci (a.k.a. $\beta$-hemolytic streptococci or BHS) according to the Lancefield grouping are classified into Groups: A, B, C, F and G. The division takes into account the presence of a specific polysaccharide antigen on the streptococcus surface [11].

Group A streptococcal infections (caused by Group A Streptococcus or GAS) pose a serious health risk to the patient, including the risk of death [11]. Group A streptococci are by far the most dominant causative organism in case of pharyngitis and tonsillitis. These strains are responsible for $15 \%$ to $30 \%$ of cases among children and $5 \%$ to $10 \%$ of cases among adults [8].

Group A streptococcal infections most often start with bacterial colonization of the upper airways or skin surface wounds. Although group A streptococci are not part of the normal bacterial flora, asymptomatic colonization with these bacteria occurs in $5-20 \%$ of the population. Individuals colonized by group A streptococci play an important role in the spread of bacteria; therefore, it is extremely important to understand the mechanisms regulating long-term asymptomatic carriage [12].

Group A streptococci occur in the form of large aggregates in the oral cavity and are tightly attached to the epithelial surface. They can hide inside non-phagocytic cells where they remain alive, protected against antibiotic treatment. It is claimed that they have the capacity to produce a biofilm. The expression profile of the virulence factors produced by the bacteria present in the biofilm differs significantly from the expression profile of the virulence factors of the bacteria directly adjacent to the tissues and not producing a biofilm. Group A streptococci are characterized by the ability to transfer genes horizontally, which contributes to the transfer of virulence factors and antibiotic resistance genes. Monitoring of the changing epidemiology of this group of bacteria is of great importance [12]. The identification of group A streptococci strains is particularly important due to the possibility of complications such as rheumatic fever, heart valve failure andglomerulonephritis [8].

S.agalactiae (Group B Streptococcus, GBS) are strains responsible for miscarriages, preterm births and neonatal infections: sepsis, pneumonia and meningitis [9].

The role of Group C Streptococcus (GCS) and Group G Streptococcus (GGS) in the pathogenesis of diseases is the subject of research [13]. The main species of Group C Streptococci in humans are: S.equisimilis, 
S.zooepidemicus, S.equi, S.dysgalactiae [14]. Most often the bacteria belonging to this genus are responsible for acute pharyngitis, acute glomerulonephritis, skin and soft tissue infections, septic arthritis, myelitis, pneumonia, and bacteremia. In pediatrics, these bacteria are the cause of pharyngitis, pneumonia, endocarditis, and meningitis. They were also isolated from blood, sinuses, peritonsillar abscesses [15].

S.dysgalactiae subspecies equisimilis (SDSE) is a normal part of the human upper respiratory tract and gastrointestinal and genitourinary tract flora, but can also colonize other areas, including the skin [11]. Diseases in humans are most often associated with the $S$. dysgalactiae equisimilis subspecies classified into group G. Similarly to group A streptococci, S.dysgalactiae can cause a number of diseases in humans, ranging from local infections such as pharyngitis, tonsillitis, pneumonia, inflammations of skin or soft tissues (wound infection, erysipelas and cellulitis), to invasive and life-threatening infections: bacteremia, necrotizing fasciitis, myelitis, meningitis, endocarditisand streptococcal toxic shock syndrome [16].

In recent years, an increasing number of infections with the etiology of Group G streptococci have been reported. Apart from SDSE, Group G streptococci include S.canis and S.intestinalis [16]. Group G streptococci occur in many animals and birds, originally identified as part of the normal flora of human skin, mouth, rhinopharynx, gastrointestinal tract and intimate areas in women [17]. Group G streptococci are responsible for pharyngitis, cellulitis, meningitis, glomerulonephritis, cardiovalvulitis (endocarditis), impetigo and sepsis [10].

Also the streptococci belonging to the group of greening coloration streptococci are of great clinical importance, e.g. S.anginosus strains. In taxonomic terms, the S.anginosus group, formerly also known as the S.milleri group, represents a subgroup of streptococci to which 3 separate species belong: S.anginosus, S.constellatus and S.intermedius [18].

The S.anginosus bacteria are commensals of human mucous membranes. They are common in the digestive tract and urinary system. S.constellatus prevails in the gastrointestinal and/or respiratory tract and S.intermedius in the head and neck area. These listed microorganisms are also known for their tendency to form abscesses with a high relapse rate, especially in the case of infections after surgical procedures, mainly in immunocompromised patients or patients with coexisting diseases, as well as in patients with implanted dental implants or dentures [18]. Moreover, S. anginosus tends to be a bacterial agent in blood infection, less responsible for abscess formation [19].

S.anginosus, unlike S.constellatus and S. intermedius, produces only a moderate amount of hexose, which is necessary for the effective formation of plaque. On the other hand, S.constellatus and S.intermedius preferentially colonize in the form of biofilms, forming plaque and usually occur in patients with periodontitis [19]. The study also showed increased susceptibility of $S$. anginosus strains to clindamycin and erythromycin. This fact is particularly important for people who cannot be treated with penicillin, considering that penicillin remains the firstline drug of choice for S. anginosus infection [18].

Other viridans streptococci - S.mutans are commensals of the oral cavity but at the same time contribute to the severity of the disease due to their ability to produce glucan and lactic acid. The expression of virulence factors varies, depending on the place and severity of infection and degree of host immunity [19].

Bacterial (dental and laryngological) complications and their interrelationships
An independent issue of medical importance is the existence of a strong relationship between sinus and laryngological diseases and dental problems. Dental problems in this relationship can be divided into three groups of diseases: dental caries complications, periodontal disease complications, peri-implant complications.

All the complications may have only inflammatory etiology, sometimes additionally traumatic-occlusal-inflammatory etiology or iatrogenic-inflammatory etiology and by ascension may affect nearby anatomical structures (e.g. teeth or implants in the lateral section of the jaw in relation to the maxillary sinus).

Untreated deep dental caries can lead to pulp inflammations, which in turn can lead to periapical infections. infections most often manifest in periostitis, the formation of abscesses, granulomas, fistulas, or periapical cysts. Decay and necrosis of the dental pulp may result in bacteria finding their way into the bloodstream (direct pathway of bacteraemia) and cause systemic complications (endocarditis, rheumatoid arthritis, sepsis).

Pulpitis is associated with complications in the root canals and related complications related to endodontic treatment. Each of the bacteria found in the oral cavity can infect the root canals. In practice, however, there is a huge difference between potential and actual bacterial flora detected in the root canals. The reason is the specificity of the intracanal environment in which biological selection determines the course and type of infection. The microbiological composition in this case is determined by the following factors: interaction between microorganisms, lack of oxygen in the environment and availability of nutrients. Research on the dynamics of root canal infections, during infections, shows a significant increase of anaerobic organisms, and a decrease in facultative anaerobes. Oxygen and oxygen products play a significant role as determinants in the development of bacterial flora. Nutrients can be supplied from the oral cavity, from degenerated connective tissue, dentinal tubules, or fluid from periapical tissues [20].

Nair was the first to identify biofilm structures in infected tooth canals [21]. He examined teeth with severe carious damage of crown tissues and periapical tissue inflammation. He observed that most of the microorganisms functioned in the form of loose groups of cocci, bacilli, filamentous bacteria and spirochetes suspended in what he considered to be a moist area of the root canal and dense colonies that were attached to dentin and formed layers of bacteria of various thickness.

Molven et al. [22], similarly to Siqueira and Lopes [23], examined the apexes of roots with periapical tissue inflammation using a scanning electron microscope. They found cocci, bacilli, filamentous bacteria and spirochetes inside the canals. Spirochetes and cocci formed the characteristic "corn-cob" formation, known primarily for its occurrence in plaque.

The latest research by Ferk Luketić et al. [24] and Ozok et al. [25] demonstrated the presence of the following mixture of bacteria in teeth restored using various methods and materials: S.mutans, S.mitis, Lactobacillus, S.aureus, E.faecalis and a biofilm mixed with Fusobacterium nucleatum and Peptostreptococcus (micromonas) micros. Despite the use of modern methods and materials for the clearing, disinfecting and closing root canals, the authors found the above-mentioned bacterial leakage around the root canal fillings. In addition, they also found the presence of Porphyromonas gingivalis, Porphyromonas endodontalis, Staphylococcus sp., Actinomyces sp., Treponema sp., Streptococcus sp., E. coli. 
The periodontium consists of root cementum, periodontal ligaments, alveolar bone and gingiva. Periodontal disease means changes in periodontal structures caused directly by plaque and independently by the cascade immune response of the host [26], which through inflammatory mediators (cytokines, metalloproteinases) leads to the loss of bone support and other periodontal structures around teeth [27]. This leads to the need of extraction of tooth becoming loosened over time and to occlusal disorders. Through the bacteria and inflammatory mediators entering the bloodstream, it can also have a significant systemic effect on patients. Periodontitis can lead to systemic inflammation and its consequences: atherosclerosis, increase in arterial pressure, increase in the mass of the left ventricle and the frequency of cardiovascular incidents, including strokes. The above-mentioned complications are the result of both direct impact of bacteria entering the bloodstream and indirect effect of bacteria. The indirect impact of bacteria includes triggering a cytokine-inflammatory cascade, mainly by lipopolysaccharides originating from the bacterial capsule and producing inflammatory mediators such as interleukin-1, $-6,-8$, tumor necrotic factor a (TNFa), prostaglandin E2, interferon, acute-phase protein (C-reactive protein or CRP). Synergic effects of the mentioned cytokines lead to the resorption of part of the alveolar bone [27]. Inflammatory mediators, by modifying the endothelial function, increasing proliferation and migration of smooth muscle cells, impairing the fibrinolysis process, and increasing the activity of plasminogen activator inhibitor, may initiate and significantly intensify the atherosclerotic process [28]. Periodontal disease independently leads to rheumatologic complications, lung diseases, kidney damage and premature births and may contribute to low birth weight [29].

A significant bilateral relationship between periodontal disease and the course of diabetes was demonstrated. Diabetes increases the risk of occurrence or exacerbation of clinical course of periodontal disease as a result of increased saliva viscosity, microangiopathy, neutrophil apoptosis defect in diabetes during exposure to bacterial lipopolysaccharides and reduction of synthesis of collagen and glycosaminoglycans in periodontal tissue fibroblasts and increased collagenase activity. Periodontal disease affects the deterioration of glycemic control and increases insulin resistance. Periodontal diseases and diabetes are significantly related, and inflammatory processes are at the root of this relationship [28].

The etiology of periodontal disease is associated with primary and secondary factors. Dental plaque is a primary factor, as a result of the qualitative and quantitative imbalance of the periodontal pocket ecosystem. Secondary factors facilitate and modulate the course of the disease (trauma factor, genetic factor, stress, alcohol, cigarettes, systemic diseases). Despite the multi-factorial etiology of periodontal disease, the primary effect of dental plaque, i.e. colonization of hard and soft tissues of the oral cavity with specific types of bacteria [30] remains the common feature, similarly to dental caries. Periodontitis is a focal point of infection and may cause inflammation of the blood vessel walls and promote the development of metastatic focal diseases. Other potential sources of infection within the oral cavity are: carious cavities with necrotic and gangrenous pulp, teeth with improper endodontic treatment, tooth roots not removed during extraction, etc. Figure 3 presents a foreign body, a fragment of the dental root after surgery with closed mucosal edema. However, the most common cause of these diseases is poor oral hygiene and the accumulation of biofilm causing periodontal diseases. Periodontitis is preceded by gingivitis. Biofilm forms an ecological niche of sorts for a large number of pathogenic bacteria. The existence of the polysaccharide barrier is the cause of resistance to antibiotics and antiseptics.
The results showed that plaque formation is the result of the process of adhesion and reproduction of bacteria, due to the existence of glycocalyx, fimbriae and the possibility of adhesion $[31,32]$. The main pathogens involved in plaque formation are the following Gram-negative and Gram-positive bacteria: Helicobacter pylori, Chlamydia pneumoniae, Mycoplasma pneumoniae, Cytomegalovirus, Porphyromonas gingivalis $(P g)$, Streptococcus sanguis, Prevotella intermedia, Aggregatibacter actinomycetemcomitans $(A a)$ [33]. Their presence is the cause of chronic periodontitis. Infection with the periodontitis pathogens (e.g. Porphyromonas gingivalis) causes vascular endothelial dysfunctions and disorders of regulation of adhesion molecules: vascular cell adhesion molecules, intercellular adhesion molecules, E-selectin and proinflammatory cytokines: interleukin 6 and interleukin 8. As the periodontitis intensifies, the number of Gramnegative bacteria as well as the amount of toxins released by them increases, and pathological pockets are formed. This is accompanied by the secretion of inflammatory mediators to gingival crevicular fluid and peripheral blood [27].

Bacteria affect the body directly (the bacteria alone) and indirectly (bacterial products). As a result of transient bacteremia, the microorganisms from periodontal pockets penetrate into the aorta as well as large and medium arteries via blood vessels. Enzymes secreted by bacteria: (hyaluronidase, collagenosis, beta-glucuronidase) break down the structures of connective tissue and epithelium. Bacterial pathogens such as $\mathrm{Aa}, \mathrm{Pg}$, E.coli cause the release of neutrophil gelatinase and methylproteinase from granules by stimulating interleukin 8 . Moreover, Streptococcus sanguis and Porphyromonas gingivalis produce the platelet aggregation initiating protein, which further provokes intravascular coagulation [34]. The formation of transient bacteremia is facilitated by daily oral hygiene activities performed by people with gingivitis or periodontal disease (e.g. brushing, flossing, chewing food) and professional dental procedures performed in dental offices (such as tartar removal, sandblasting, polishing, probing).

The significance of bacterial plaque in the etiology of dental caries alone has been known for a long time and has been documented in numerous studies [35-39].

In the pathogenesis of dental caries, the ability of Streptococcus mutans to survive in low $\mathrm{pH}$ conditions is an important virulence factor. This bacteria species is both acid-resistant and acid-forming, i.e. capable of metabolizing sugars in conditions of prolonged $\mathrm{pH}$ decrease and even is well-prepared for growth in such a difficult environment [40]. This is not the only property, however, of bacteria associated with dental caries. Compared with other microorganisms, these bacteria are able to quickly transport fermentable sugars inside the cell and transform them into acids damaging the hard tissues of teeth [40]. They also produce extra- and intracellular carbohydrates, which participate in the plaque matrix formation, or are the backup substances used for energy generation or transformation into acids in the case of limited access to sugars [37]. There are numerous species of Gram-positive streptococci present in dental plaque which have acid-forming and acid-resistant properties and contribute to the development of dental caries, e.g. S.salivarius, S.mitis, Lactobacillus. However, S.mutans is considered to be the main bacteria species causing dental caries, which is confirmed by the presence of this streptococcus strain in a large number of teeth affected by caries [41]. The research also proves that it is possible that despite the high bacterial index of mutans streptococci group in dental plaque no demineralization of enamel is present or no carious cavities are visible. This is most probably due to the presence of other species of bacteria in dental plaque, which - as a result of 
transformations - produce substances raising the $\mathrm{pH}$ of the plaque, e.g. ammonia produced by $S$. salivarius from urea or arginine by $S$. sanguinis or the presence of Veillonella consuming lactic acid produced by streptococci $[38,39,41,42]$.

In the caries process, the microflora and susceptible tooth tissues influence each other through frequent consumption of fermentable carbohydrates or changes in saliva secretion by the host. The multifactorial nature of the dental caries disease process causes caries to occur as a result of disturbing the natural balance of the normal microflora of the biofilm, i.e. dental plaque [43]. This poses a significant health threat not only to the healthy surfaces of hard dental tissues (enamel, dentin, root cementum), but also results in repeated failures of the dental treatment undertaken. The literature describes the presence of bacterial biofilm inside root canals and also on the external surface of roots [39]. The results suggest that biofilm may be associated with failed endodontic treatments (inadequate root canal filling), persistent periapical tissue inflammation. Biofilm is also present on many restorative materials (composites, glass ionomer cements), prosthetic restorations, dental braces, and is also responsible for secondary caries (observed at the edge of the existing restoration).

Dental plaque, resulting from poor hygiene, also plays an important role in the pathogenesis of peri-implant infective diseases (PIIDs) commonly known as peri-implant mucositis (PIM) and periimplantitis (PI). Mucositis is reversible and restricted to the peri-implant mucosa. Periimplantitis is an inflammatory lesion in the gingiva and affects

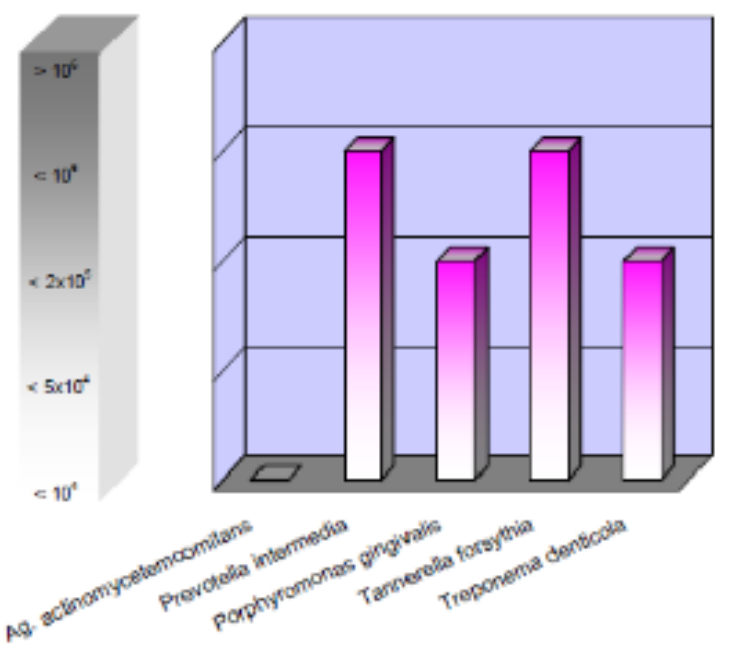

implant-supporting bone. The most common cause of PI is the anaerobic Gram-negative bacteria (e.g.: Actinobacillus, Campylobacter spp., Fusobacterium nucleatum, Porphyromonas gingivalis) having a chronic effect on the subgingival area, which, in combination with inflammatory mediators resulting from the host immune response, lead to tissue loss around the implants. The same bacteria can be observed in periodontal pockets , therefore periodontal disease has a significant impact on development of peri-implant infective diseases (PIIDs) Figure 7 shows an increase in the same bacterial strains (PCR-DNA molecularbiological test for detection of periodontitis -associated microorganisms) in the perio-pockets as around implants inserted in a patient with periodontal disease. Figure 8 shows radiographic changes (OPT) in the same patient diagnosed with periodontal disease and periimplantitis.

If the tissue loss in PI is significant, this ultimately and finally leads to the necessity to remove the infected implant [44]. The prevalence for PIM was reported to be up to $80 \%$ and for PI about $56 \%$ Early infections can usually be observed as a result of impaired wound healing, late infection after process of implant integration. Inflammatory bone infiltration around the implants may involve anatomical structures accompanying the lesions, and in the case of lateral sections of the jaw it may affect the maxillary sinuses. Peri-implant complications may be associated with incorrect placement of implants (inadequate prostheticsurgical planning), occlusion disorders, iatrogenic bone overheating or iatrogenic crown cementation (cementitis), and finally with material abnormalities (e.g. broken prosthetic abutments) or other risk factors

igure 7. Comparison of PCR-DNA molecularbiological tests for detection bacteria from periodontal pockets and around implants with coexisting periodontitis and periimplantitis

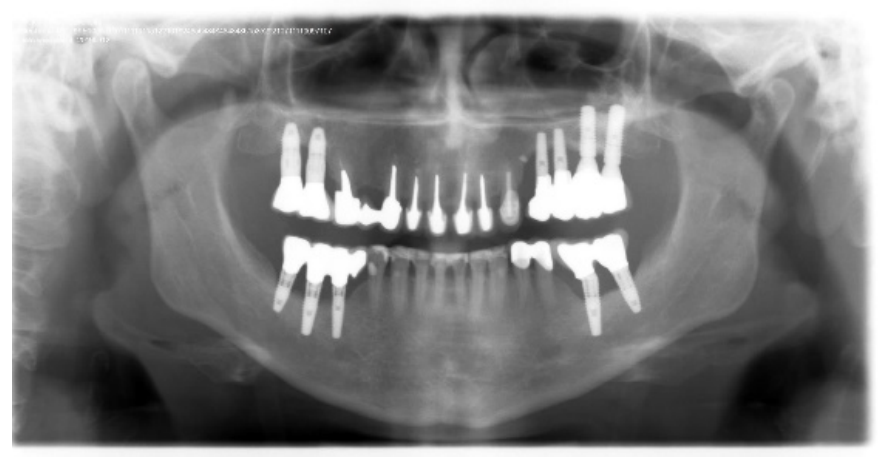

Figure 8. Radiographic (OPT) visualization of co-existing periodontitis and periimplantitis 
(alcohol, cigarettes, systemic diseases) [45,46]. Reactive sinusitis after implantological treatment is shown in Figure 5. Examples of sinus inflammation with foreign bodies, most often tooth root fragments and endodontic materials, are shown in Figures 1,2,3 and 4.

Most patients with oral cavity bacterial infectious diseases postpone their visits to specialists. Sometimes such delays may pose a threat to health and life, especially in patients with high fever, impaired respiratory function, and improper nutrition due to pain when biting down, limited mouth opening, chewing and swallowing disorders [19].

Periimplantitis are most commonly caused by Gram-negative bacteria containing lipopolysaccharide (LPS) in their cell walls [45]. It has been postulated that LPS is the key factor in the development of periimplantitis and bone resorption due to bacterial complications [47].

The conservative treatment of periimplantitis includes (similarly to periodontitis) mechanical cleaning (scaling), pharmacological treatment (antiseptics and antibiotic therapy) and laser therapy (erbium and diode lasers) [45]. The lasers used, apart from the removal of bacterial deposits and tartar from the implant and decontaminating and antiseptic action, reducing inflammatory infiltrate, may also weaken the LPS-induced inflammatory response [47]. Removal of the bacterial biological layer and all deposits around the implants, professional and home hygiene (carried out by patients themselves), seem to be of key importance in the prevention and treatment of periimplantitis. The basic line of defense is to inhibit the initial stage of bacterial adhesion to the implant surface [48]. Preventing the development of bacteria on the surface of implants requires perfect oral hygiene both by the patient (methods of brushing, flossing) and through visits to a dental hygienist and dentist's offices (at least once every 6 months). Additionally, appropriate antiseptics should be used. The development of new technologies also includes the application of antimicrobial coatings of implants. Innovative nanocoatings allow avoiding the administration of antibiotics [44].

Preventing dental caries, periodontal diseases, periimplantitis (as odontogenic factors of further complications in the facial skeleton area) is done by preventing the development and accumulation of bacterial biofilm. In preventing the formation of bacterial biofilm, the primary - and at the same time the simplest - method is home and professional oral hygiene based on regular cleaning of teeth, prosthetic restorations and orthodontic appliances appropriate for a given person with the use of suitable devices, tools and dental agents. However, home oral hygiene based on flossing and tooth-brushing alone is often not enough. Mechanical removal of dental plaque must be supported by the use of mouthwash containing biofilm inhibitors and the use of e.g. sugar-free chewing gums as an auxiliary hygienic agent [48-50]. Among the most commonly used additives to mouthwashes and toothpastes are agents with antibacterial properties, which demonstrate chemical stability and therefore have a long-lasting effect in the oral cavity environment of patients, inhibiting the adhesion of plaque and related development of streptococci. These include chlorhexidine, triclosan, sanguinarine, zinc salts and fluorine, which increases the resistance of enamel to acids produced by bacteria associated with dental caries. In many cases, diluted hydrogen peroxide is also recommended due to its wide spectrum of activity. Hygiene alone is not always sufficient to eliminate the effects of bacteria in the oral cavity of patients [40]. New material technologies eliminating polymerization shrinkage of materials (marginal leakage facilitates accumulation of dental plaque) are enriched with factors inhibiting bacterial growth (compomers, glass ionomers, cements contain currently fluorine compounds).
As the literature shows another solution to prevent the development of biofilm in the oral cavity is the use of nanosilver in dental materials [40,51-54]. Numerous studies have confirmed that materials and medications containing nanosilver have very effective antimicrobial effects $[51,52,55]$. The antibacterial properties of silver have been used in medicine for many years. In dentistry, silver is used in materials used to fill cavities (main component of amalgam fillings), as well as to impregnate carious tooth tissues in children (using 10-25\% silver nitrate). Nanosilver is also used in systems bonding composite fillings with tooth tissues (primer, bond) and in addition nanosilver gels is used in prosthetics (protect pillar teeth prepared for prosthetic restoration).

In in vitro and in vivo studies, a long-term inhibitory effect against S.mutans and Lactobacillus while maintaining favourable mechanical properties of composites enriched with silver ions was achieved $[42,48,56]$. Yoshida et al. demonstrated the antibacterial properties of composite resins containing high concentrations of silver nanoparticles (about 10\%) [48]. Kasraei et al. proved that 1\% concentration of nanosilver added to composites inhibits the development of bacteria associated with dental caries [56].

Numerous studies confirmed the validity of the use of nanosilver in endodontic treatment. Preparations with nanoparticles kill anaerobic bacteria (often responsible for endodontic treatment failures) [54,57]. Alcoholic nanosilver solution (used for rinsing root canals before final filling) was shown to be highly effective in eliminating $E$. faecalis from infected canals. The effectiveness of nanosilver in endodontic treatment led to further, more advanced research $[57,58]$.

Nanosilver used in dentistry significantly reduces the viability, metabolic activity and count of plaque-forming bacteria, which reduces the production of lactic acid in the biofilm [40].

Proper oral hygiene and related pharmacological measures prevent the adhesion of bacteria to the surface, affect the communication mechanisms of microorganisms, inhibit the decomposition of sugars (with the use of metabolic inhibitors and non-fermentable artificial sweeteners). Moreover, pharmacological or mechanical stimulation of saliva flow in the patient's oral cavity and reinforcement of host defence mechanisms may help maintain homeostasis of bacterial plaque [40].

\section{Discussion}

Oral cavity bacteria have been known for many decades and are of constant interest to dentists, patients and general doctors. Oral cavity microbiota is extremely diverse and includes over 700 species of dental plaque-forming bacteria. Dental plaque is an example of one of the best-known biofilms formed by the layered growth of microorganisms capable of adhering to each other and organized in microcolonies [41,55,59-61].

Microorganisms found in the oral cavity are found around the teeth in the form of two types of biofilm: supragingival and subgingival, which differ significantly from each other in terms of composition of the bacterial flora. The supragingival plaque is dominated by Gram-positive streptococci such as S. mutans, S. salivarius, S. mitis and Lactobacillus, while the subgingival plaque is dominated by Gram-negative anaerobic bacteria such as Actinobacillus, Campylobacter sp., Fusobacterium nucleatum, Porphyromonas gingivalis. Dental caries is usually caused by supragingival biofilm, whereas subgingival biofilm is associated with gingivitis and, consequently, periodontal diseases, as well as periimplant complications $[35,62]$. In addition to the above-mentioned bacteria, E. faecalis, S. aureus, E. coli, Porphyromonas endodontalis, 
Staphylococcus sp., Actinomyces sp., Treponema sp. and Streptococcus $s p$., play an important role in peri-implant and endodontic treatment complications.

The bacterial flora present in the biofilm is characterized by high variability. There are specific areas: mouth, tongue, palate, cheeks, teeth and gingival pockets, which have their own specific microflora [29]. According to Smith et al. specific bacteria prefer specific oral colonization sites, and their characteristics allow for the division into primary and secondary colonizing bacteria. Bacteria belonging to the Streptococcus genus are considered the primary colonizers [63].

The relative number of commensal oral cavity species is quite stable in healthy individuals and shifts in these proportions are likely to indicate disease activity [64]. The imbalance between the number of pathogenic and non-pathogenic bacteria in the oral cavity microflora contributes to more frequent adhesion of bacteria to the surface and thus increases the risk of periodontal infections; it is also often the causative factor of peri-implant oral mucositis [30].

According to reports from 2008, staphylococci and streptococci are the most common microorganisms in intracranial complications. The infiltration of frontal sinuses and presence of anaerobic bacteria and multi-bacterial infections are the factors determining the more severe course of infections, sometimes the need for surgical interventions, prolonged intravenous antibiotic treatment and longer hospitalization [65].

In the 1990s it was reported that the incidence of intracranial complications in children with paranasal sinusitis was 3.7\%. More recent studies have shown that orbital and intracranial complications may affect as much as $3-5 \%$ of hospitalized patients with sinusitis [65].

The results of microbiological tests carried out in patients with odontogenic infections indicate the dominance of Gram-positive streptococcus strains and Gram-negative strains of Bacteroides and Prevotella. Streptococci and anaerobic bacteria are the main microbiological agent in the primary infection and E.faecalis strains - in the secondary infection. However, there is no single accepted protocol of standard antibacterial procedure in the treatment of odontogenic infections [44].

Examples of facial skeleton complications related to endodontic and implantological treatment are presented in Figures 2-5.

Examples of fungal masses formed after endodontic treatment are presented in Figure 6.

So far, only a few cases of meningitis with GCS etiology have been described in the literature. Clarke et al. first presented a case of the disease in the presence of cavernous sinus thrombosis. A possible complication of meningitis may be the development of cavernous sinus thrombosis. Cavernous sinus is a cavity at the base of the brain that drains oxygenated blood from the brain to the heart. The infection is usually caused by the spread of infection from the nose, sinuses, ears or teeth. Cavernous sinus thrombosis is a life-threatening infection that can lead to permanent neurological consequences. Earlier pharyngitis may be an important risk factor [14].

Subramani et al. were the first to report a rare case of brain abscess caused by Group G Streptococcus in a 12-year-old child. Brain abscess is a serious, life-threatening infection. The most common source of infection that contributes to brain abscess is paranasal sinusitis, otitis media or dental infections. Another possible source of infection is the bloodborne spread of bacteria from a distant non-cranial focus. Important predisposing factors are: otitis media, past craniotomy and congenital heart defects. The examined girl was diagnosed with congenital heart defects at birth. The patient with neurological symptoms was treated with ciprofloxacin and amoxicillin with clavulanic acid [66].

Szyfter et al. have discussed the matter of microbiology of cerebral abscesses, including empyemas. Surgical treatment of complications was preceded by empirical antibiotic therapy. The secretions from abscesses were tested by means of the bacterial culture method. Microbiological analysis showed mainly the presence of Grampositive bacteria (streptococci and staphylococci). Anaerobic strains (Pseudomonas, Propionibacterium, Bacteroides and Fusobacterium) dominated in extradural and subdural empyema. Only in $30 \%$ of cases a correlation between the bacterial flora of the paranasal sinus and abscesses was found. In about $30 \%$ of the abscesses no bacteria were cultured. The results of microbiological tests allowed the use of targeted antibiotic therapies [67].

Streptococcus strains predominate also in the case of peritonsillar abscesses. A peritonsillar abscess is a purulent complication of acute tonsillitis. It most commonly concerns teenagers and young adults. There are many inconsistencies with regard to the microbiology of abscesses. The etiology of most abscesses is a multibacterial infection. Three pathogens play a key role here: S. pyogenes, Fusobacterium necrophorum and strains of the $S$. miller igroup.

Mazur et al. observed bacterial growth in 9 out of 45 cultures of aerobic materials taken from peritonsillar abscesses (20.0\%). In 15 oxygen cultures $(33.3 \%)$ only species belonging to the normal throat bacterial flora, namely S.mitis, S.oralis, S.salivarius, S.sanguinis and other streptococci, excluding those belonging to the S.milleri group, were detected. Potential pathogens were identified in 29 oxygen cultures (64.4\%). The most frequently isolated pathogen was S.pyogenes, found in 13 out of 45 oxygen cultures (28.9\%). It was most often isolated as the only strain $(10 / 13,76.9 \%)$. In three cultures S.pyogenes was detected as one of two isolates, together with Enterococcus sp., Escherichia coli and S.salivarius, respectively. Of the 45 patients, whose abscess aspirates were cultured, 25 (55.6\%) received antibiotic therapy before admission. Differences in isolation of potential pathogens were observed between patients with and without prior antibiotic therapy, although they were not statistically significant. Potential pathogens were isolated from patients who did not receive any antibiotic therapy before admission more frequently than normal bacterial throat flora. Moreover, when S.pyogenes was analysed separately from other potential pathogens, it turned out that its isolates were much more frequently observed in patients without prior antibiotic therapy [68]. Other researchers have observed different relationships. Megalamani et al. [69] confirmed that the percentage of isolation of S.pyogenes strains in patients without prior antibiotic treatment was similar to that observed in patients who were treated with antibiotics before the occurrence of an abscess.

Hong et al. conducted a cross-sectional test of bacterial microflora using pyrosequencing based on the 16S rRNA gene. Samples were taken from an 8-year-old boy with obstructive sleep apnea and chronic otitis media with effusion. The material came from the middle ear, the palatine tonsils and the pharyngeal tonsil. Pseudomonas sp., strains predominated the middle ear $(82.7 \%)$, while streptococci predominated the tonsil microbiota (69.2\%). Pharyngeal tonsil microbiota included bacteria detected both in the middle ear and the palatine tonsils. The dominant strains were Pseudomonas sp., Streptococcus sp., Fusobacterium sp., and Pasteurellaceae bacteria. The authors concluded that the tissue of the pharyngeal tonsil may be a source of pathogenic bacteria for both middle ear and palatine tonsils infections [70]. 
In the study of patients with CS in the British population, the dominant microbiological factor was $S$. anginosus bacteria causing acute bacterial inflammation of the nasal and sinus mucosa (61\%) and orbital inflammation (83\%) and/or intracranial abscesses (78\%). In all cases, these organisms were susceptible to penicillin. According to the authors, the incidence of $S$. anginosus strains is much higher than previously thought. In 1998 microbiological results of sinusitis in developing countries were presented, isolates of S. anginosus in $50 \%$ of abscesses were reported. In 1981 S. anginosus strains were the second most common pathogen (38\%) in acute sinusitis. A significant portion of patients (42\%) had mixed infections [4]. Also, Mortimore et al. [71], on the basis of data from the Republic of South Africa, confirmed that the most common microorganism cultured in the case of orbital or intracranial complications of sinusitis was $S$. anginosus. These results are also consistent with the research carried out by Rudloe et al. [72]. Viridans streptococci, including S.anginosus, can remain in synergistic relationships with anaerobic bacteria of the oral cavity, which increases their virulence. This is observed in complications of sinusitis, among others. The question remains whether improper oral hygiene is a risk factor for the development of acute sinusitis complications by promoting the growth of $S$. anginosus organisms in synergy with anaerobic bacteria [73].

Alanzi et al. examined Streptococcus strains isolated from dental prostheses of people with cancer problem $(n=11)$ and without cancer $(n=15)$, as well as from the oral cavity of healthy people $(n=20)$. The authors proved that the ability of adhesion to the cheek cell membrane was much higher among S.mutans isolates isolated from denture patients and cancer patients with dental prostheses, as compared to healthy individuals. Adhesion of microbial cells to the surface of the host mucosa is essential for colonization and further development of infection. S.mutans strains use their receptors to adhere to the surface of the teeth and have the ability to form a biofilm. Increased lactic acid production facilitates cell proliferation and thus promotes the development of cancer. In both groups of patients, the microorganisms showed increased potential to initiate infections and the infections were more severe and prolonged [19].

Bacterial adhesion on dental implant-based suprastructures is the main cause of peri-implant complications. A meta-analysis carried out by Meza-Siccha et al. showed that the incidence of inflammations in the implant area is $29,48 \%$. The results showed that S.mutans strains are an important colonizing factor as they adhere to titanium and zirconium surfaces. S.sanguinis strains dominated zirconium implants. The viability of bacteria was similar in both types of materials [30].

Infectious endocarditis is a debilitating disease with high morbidity and mortality. The factors involved in the development of bacterial endocarditis are difficult to define. Two elements seem to be decisive: the surface of endocardium damage and high bacterial load in the blood. The main microorganisms responsible for the disease include staphylococci, streptococci, and enterococci, which are responsible for up to $90 \%$ of cases. Streptococci present in the oral cavity (S.mutans and S.sanguinis), present in dental plaque, may enter the bloodstream during mucosal damage, causing bacteremia and spread of the infection in places distant from the original source of infection [29]. Eykyn et al. confirmed that most, if not all cases of streptococcal endocarditis are caused by poor oral cavity hygiene (and the related dental caries and periodontal diseases) rather than by dental procedures [74].

Preventing the development of dental caries, gingivitis and, consequently, periodontal disease contributes to reducing the number of cases of infectious endocarditis of bacterial etiology involving oral cavity microflora. For this purpose, it is necessary to improve home and professional oral hygiene of patients and to observe dental check-up visits (at least once every 6 months), remove sources of infection (caries, untreated teeth), and in case of periodontal disease - professional three-stage periodontal treatment. Only check-up and therapeutic visits as well as conscious cooperation of the patient with regard to hygiene can reduce or eliminate inflammatory processes in the oral cavity, which should decrease the incidence of bacteremia and related systemic complications (cardiovascular diseases, atherosclerosis, infarcts, strokes, endocarditis, kidney and lung diseases) and dental complications (the need to extract teeth due to periodontal diseases and caries) [29].

Mandibular bone inflammation is not a common inflammation. The frequency of diagnosis of the disease has dramatically decreased in the last 50 years after the introduction of antibiotics and improvement of medical and dental care. Most often we deal with odontogenic infections, followed by secondary infections associated with craniofacial fractures and the presence of foreign bodies (e.g. endodontic materials, left apexes of roots, dental implants, plates, screws). Pre-existing diseases such as chronic systemic diseases, alcoholism, immunosuppression, malnutrition, diabetes, drug abuse, cancer, osteopetrosis, Paget's disease, radiotherapy may be associated with an increased frequency of osteomyelitis. Although different micro-organisms present in the oral cavity in healthy individuals may potentially be involved in the occurrence of disease, no particular micro-organism has so far been identified as the dominant etiological agent. There is often talk of a multi-bacterial infection [18].

So far, 2 cases of development of bacterial osteomyelitis after implantation of a dental implant associated with S. anginosus infection have been reported Chetalain et al. presented another such case. The patient developed mandibular osteitis with S.anginosusetiology after insertion of a dental implant. The aim of this study was to estimate the possible risk factors of the disease [18].

An infection with S.anginosus etiology is associated with an increased risk of surgical procedures and prolonged antibiotherapy Chetalain et al. noted the need for radical surgical reconstructive procedures (two thirds of cases), prolonged antibiotherapy (mean of 9 months) and bacterial resistance to penicillin (two thirds of cases). The study showed that mandibular osteitis with S.anginosus etiology is a particularly aggressive form of osteomyelitis, with a tendency to develop in women aged 60, who are smokers or show the presence of accompanying diseases affecting bone metabolism. The authors recommend weekly check-ups during the first 3 months after surgery and insertion of a dental implant [18].

Streptococci may contribute to orbital complications. Orbital cellulitis may be caused by primary sinus, skin or dental infections. Almost two thirds of the cases, $64 \%$, come from a primary sinus infection, which is the most common cause of orbital inflammation, most of which is characterized by bacterial etiology. $16 \%$ of cases originate from skin lesions such as eczema, boils or cellulitis of the face. The most common manner of spreading the infection are paranasal sinuses [75].

Infections in the orbital area with S.constellatus etiology are rare, but may occur, especially in immunocompromised patients, in individuals with coexisting odontogenic infections and rapidly progressing necrosis. Early diagnosis enables rapid surgical intervention (cleaning) to eliminate infection. Moreover, it protects against permanent loss of sight, damage to the surrounding structures and sometimes death 
[76]. Two cases of secondary orbital cellulitis in S.constellatus infections have been reported. One case was associated with prolonged cavernous sinus thrombosis, the other was associated with an upper molar infection. In both cases the bacteria responsible for orbital infections showed resistance to intravenous antibiotics and eventually surgical intervention was necessary. In the second case, visual acuity was reduced and only included the ability to see light [76].

Li et al. presented a case of orbital cellulitis caused by odontogenic S.constellatus strains [36]. In comparison to the previously described reports, the case described was exceptional as it was the only described example of an odontogenic infection successfully treated without removing the eyeball. The patient received conservative surgical cleansing and intravenous antibiotic therapy. The patient regained full extraocular mobility but did not regain sight. Her visual limitations were probably due to visual nerve ischemia, secondary to a widespread infection [76].

An interesting case of S.constellatus etiology infection was described by Allegrini et al. [75]. In the report presented, the source of the disease was unusual: a dental infection. The S.constellatus strain was isolated from the blood of a patient, who was confirmed to have a jaw abscess. The patient also suffered from co-occurrence of cavernous sinus thrombosis and pulmonary embolism, which further worsened his health. The difficulty in diagnosing the presented case was connected to the fact that the patient did not manifest odontogenic problems and did not use immunosuppressive therapies.

The presented report illustrates the necessity of quick diagnosis and treatment of orbital cellulitis with bacterial etiology. It is important to properly identify the causes of orbital cellulitis and systemic complications. Infection can lead to abscesses, blindness, sometimes death and is usually a consequence of bacterial sinusitis. In the presented case, only ophthalmic symptoms were observed in the patient. A multidisciplinary diagnostic and therapeutic approach have made it possible to quickly make a proper diagnosis and implement treatment covering both the main disease as well as the accompanying cavernous sinus thrombosis and pulmonary embolism. This probably saved the patient from serious and permanent consequences such as brain ischemia and sepsis. The authors stress the need for an interdisciplinary approach and radiological support in the diagnosis and treatment of orbital cellulitis. Sometimes, the presence of a disease can be the first indication of the spread of infection in the body [75].

\section{Conclusion}

Invasive streptococcus infections apart the undoubted heavy burden for patients also remain a major challenge for clinicians. Knowledge of the properties of virulence factors, their regulation and role can help clarify the relationship between the bacteria and the host. Side effects associated with the use of long-term antibiotic therapy in the treatment of bacterial inflammatory diseases, such as growing antibiotic resistance of the strains remain a big problem for current medicine. The economic factor associated with treating severe infections is also important. In this context prevention is the most important solution.

Epidemiological and microbiological surveillance is necessary to monitor the development of streptococcal infections and to develop better prevention strategies.

Streptococci are often treated as commensal bacteria and their pathogenicity is underestimated. Commensal bacteria in predisposed individuals with a weakened immune system can contribute to serious complications covering different areas of the body: from facial skeleton complications to septic arthritis, endocarditis, or sepsis. Therefore, early and proper diagnosis is very important as well as initiating targeted treatment in cases of both laryngological diseases and dental infections. The incidence of bacteraemia following many dental procedures such as scaling, root planning, periodontal surgery, endodontic treatment under normal circumstances (good oral health and dental care) is fought by the right balance of barrier systems of oral cavity working together with host immune response. When oral hygiene is poor the numbers of bacteria, colonizing teeth supra and subgingivally, could increase two to even ten-fold. This increases the risk of microbes penetrating into the tissues and to the bloodstream inducing bacteraemia. For the above reasons the most important thing is, as always, prevention. Proper oral hygiene regime is often the key to success just like diets, good eating habits and no risk factors (alcohol, cigarettes, stress). Continuous contact of oral cavity with the external environment results in diversity in the composition of the oral microbiome. Diet, temperatures, $\mathrm{pH}$ of saliva, feeding habits are very important factors that contribute in the establishment of oral microbiota. Unbalanced microflora affects development of various diseases like tooth caries, periodontal diseases, type 2 diabetes, heart related disorders, cardiovascular problems. That is why, described in this article, factors and methods influencing on the homeostasis bacteria in biofilm as well as remodelling of microbial metabolic and physiological activities are so important for maintenance of overall health of patients. Conclusions are consistent with what someone once said and was already cited: "mouth is the gateway to the total body wellness, thus oral microbiome influences overall health of an individual".

\section{References}

1. Miah MS, Nix P, Koukkoullis A, Sandoe J (2016) Microbial causes of complicated acute bacterial rhinosinusitis and implications for empirical antimicrobial therapy. $J$ Laryngol Otol 130: 169-175. [Crossref]

2. Meltzer EO, Hamilos DL, Hadley JA, Lanza DC, Marple BF et al, (2004) Rhinosinusitis: Establishing definitions for clinical research and patient care. J Allergy Clin Immunol 114: $155-212$.

3. Michalik M(2019) Bacterial flora in chronic sinusitis (Bacterial flora in chronicsinusitis) Practical Dentistry Forum p. 44

4. Biswas K, Wagner Mackenzie B, Waldvogel-Thurlow S, Middleditch M, Jullig M, et al. (2017) Differentially regulated host proteins associated with chronic rhinosinusitis are correlated with the sinonasal microbiome. Front Cell Infect Microbiol 7: 504 [Crossref]

5. Anderson M, Stokken J, Sanford T, Aurora R, Sindwani R (2016) A systematic review of the sinonasal microbiome in chronic Rhinosinusitis. Am J Rhinol Allergy 30: 161166. [Crossref]

6. Stevens WW, Lee RJ, Schleimer RP, Cohen NA (2015) Chronic rhinosinusitis pathogenesis, J Allergy Clin Immunol 136: 1442-1453. [Crossref]

7. Anderson Eloy J, Govindaraj S (2008) Microbiology and Immunology of Rhinosinusitis Rhinosinusitis pp. 1-12.

8. Chan TV (2010) The Patient with Sore Throat. Med Clin North Am 94: 923-943. [Crossref]

9. Nobbs AH, Jenkinson HF, Everett DB (2015) Generic determinants of Streptococcus colonization and infection. Infect Genet Evol 33: 361-70. [Crossref]

10. Ilakkiya A, Parveen S, Kumar CN, Swathi S (2015) Macrolide resistance in Streptococcus species. J Pharm Bioallied Sci pp. S26-31.

11. Parks T, Barrett L, Jones N (2015) Invasive streptococcal disease: a review for clinicians. Br Med Bull 115: 77-89. [Crossref]

12. Marks LR, Mashburn-Warren L, Federle MJ, Hakansson AP (2014) Streptococcus pyogenes biofilm growth in vitro and in vivo and its role in colonization, virulence, and genetic exchange. J Infect Dis 210: 25-34. [Crossref]

13. Naik TB, Nadagir SD, Biradar A (2016) Prevalence of Beta-hemolytic Streptococc Groups A, C, and G in patients with acute pharyngitis. $J$ Lab Physicians 8: 45-49. [Crossref] 
14. Clarke M, Enuh H, Saverimuttu J, Nfonoyim J (2013) Streptococcus group C meningitis with cavernous sinus thrombosis. Infect Drug Resist 6: 79-81. [Crossref]

15. Chandnani HK, Jain R, Patamasucon P (2015) Group C Streptococcus causing rheumatic heart disease in a child. J Emerg Med 49: 12-14. [Crossref]

16. Wang X, Zhang X, Zong Z (2015) Genome sequence and virulence factors of a group $\mathrm{G}$ Streptococcus dysgalactiae subsp. equisimilis strain with a new element carrying erm (B). Scientific Reports 6: 20389.

17. Deng W, Farricielli L (2014) Group G streptococcal sepsis, septic arthritis and myositis in a patient with severe oral ulcerations. BMJ Case Rep [Crossref]

18. Chatelain S, Lombardi T, Scolozzi P (2018) Streptococcus anginosus dental implantrelated osteomyelitis of the jaws: An insidious and calamitous entity. J Oral Maxillofac Surg 76: 1187-1193. [Crossref]

19. Alanazi SAS, Alduaiji KTA, Shetty B, Alrashedi HA, Acharya BLG, et al. (2018) Pathogenic features of Streptococcus mutans isolated from dental prosthesis patients and diagnosed cancer patients with dental prosthesis. MicrobPathog 116: 356-361.

20. Figdor D, Sundqvist G (2007) A big role for the very small - understanding the endodontic microbial flora. Aust Dent J 52: 38-51. [Crossref]

21. Nair PNR (1987) Light and electron microscopic studies of root canal flora and periapical lesions. J Endod 13: 29-39. [Crossref]

22. Molven O, Olsen I, Kerekes K (1991) Scanning electron microscopy of bacteria in the apical part of root canals in permanent teeth with periapical lesions. Endod Dent Traumatol 7: 226-229. [Crossref]

23. Siqueira JF Jr., Lopes HP (2001) Bacteria on the apical root surfaces of untreated teeth with periradicular lesions: a scanning electron micro-scopy study. Int Endod J 34: 216220. [Crossref]

24. Ferk Luketić S, Malcić A, Jukić S, Anić I, Segović S, et al. (2008) Coronal microleakage of two root-end filling materials using a poly -microbial marker. J Endod 34: 201-203. [Crossref]

25. Ozok AR, Wu MK, Luppens SB, Wesselink PR (2007) Comparison of growth and susceptibility to sodium hypochlorite of mono- and dual-species biofilms of Fusobacterium nucleatum and Peptostreptococcus (micromonas) micros. $J$ Endod 33: 819-822.

26. Górska R, Laskus-Perendyk A, Gregorek H, Kowalski J (2005) The influence of surgical treatment of periodontal disase on selected lymphocyte subpopulations important for cellular and humoral immune responses. J Perodontol 76: 1304-10. [Crossref]

27. Górska R, Gregorek H, Kowalski J, Laskus-Perendyk A, Syczeska M, et al. (2003) Relationship between clinical parameters and cytokine profiles in inflamed gingival tissue and serum samples from patients with chronic periodontitis. J Clin Periodontol 30: 1046-1052. [Crossref]

28. Wożakowska-Kapłon B, Filipiak K, Opolski G, Górska R (2009) A relationship between periodontal diseases, diabetes mellitus and diabetic nephropathy. Diabet Prakt 2: $72-75$.

29. Carinci F, Martinelli M, Contaldo M, Santoro R, Pezzetti F, et al. (2018) Focus on periodontal disease and development of endocarditis. J Biol Regul Homeost Agents 32: 143-147. [Crossref]

30. Meza-Siccha AS, Aguilar-Luis MA, Silva-Caso W, Mazulis F, Barragan-Salazar C (2019) In Vitro evaluation of bacterial adhesion and bacterial viability of Streptococcus mutans, Streptococcus sanguinis and Porphyromonasgingivalis on the abutment surface of titanium and zirconium dental implants. Int J Dent 2019: 4292976. [Crossref]

31. Goteiner D, Ashmen R, Lehrman N, Janal MN, Eskin B (2008) Presence and significance of interleukin-1 polymorphism in patients who present with acute coronary syndrome, angina and epidemiologic pilot study. Il-1 and acute coronary syndrome. $J$ Periodontal 79: 138-143. [Crossref]

32. Fardi A, Papadimitriou D (2007) Periodontal and atherosclerosis-induced diseases. Systematic reviews. Int Angiol 26: 197-205. [Crossref]

33. Couper DJ, Beck JD, Falkner KL, Graham SP, Grossi SG, et al. (2008) The Periodontitis And Vascular Events (PAVE) pilot study: recruitment, retention and community care controls. The Periodontitis and Vascular Events (PAVE) pilot study. J Periodontol 79: 80-89. [Crossref]

34. Restaino CG, Chaparro A, Valenzuela MA, et al, (2007) Stimulatory response of neutrophils from periodontitis patients with periodontal pathogens. Neutrophile response in periodontitis patients. Oral Dis 13: 474-481. [Crossref]

35. Abusleme L, Dupuy AK, Dutzan N, Silva N, Burleson JA, et al. (2013) The subgingival microbiome in health and periodontitis and its relationship with community biomass and inflammation. ISME J 7: 1016-1025. [Crossref]
36. Aparna MS, Yadav S (2008) Biofilms: microbes and disease. Braz J Infect Dis 12: 526-530. [Crossref]

37. Loesche WJ (1986) Role of Streptococcus mutans in human dental decay. Microbiol Rev 50: 353-380. [Crossref]

38. Marsh PD (1994) Microbial ecology of dental plaque and its significance in health and disease. Adv Dent Res 8: 263-271. [Crossref]

39. Marsh PD (2010) Microbiology of dental plaque biofilms and their role in oral health and caries. Dental Clin North Am 54: 441-454. [Crossref]

40. Chałas R, Wójcik-Chęcińska I, Woźniak M, Grzonka J, Święszkowski W, et al. (2015) Dental plaque as a biofilm - a risk in oral cavity and methods to prevent. PostepyHig Med Dosw (online) 69: 1140-1148. [Crossref]

41. Marsh PD (2006) Dental plaque as a biofilm and a microbial community - implications for health and disease. BMC Oral Health 6: S14.

42. Seneviratne CJ, Zhang CF, Samaranayake LP (2011) Dental plaque biofilm in oral health and disease. Chin J Dent Res 14: 87-94. [Crossref]

43. Emmanuel R, Palanisamy S, Chen SM, Chelladurai K, Padmavathy S, et al. (2015) Antimicrobial efficacy of green synthesized drug blended silver nanoparticles against dental caries and periodontal disease causing microorganisms. Mater Sci Eng C Mater Biol Appl 56: 374-379. [Crossref]

44. Baghdan E, Raschpichler M, Lutfi W, Pinnapireddy SR, Pourasghar M, et al. (2019) Nano spray dried antibacterial coatings for dental implants. Eur J Pharm Biopharm 139: $59-67$

45. Kuo HN, Mei HI, Liu TK, Liu TY, Lo LJ, et al. (2017) In Vitro laser treatment platform construction with dental implant thread surface on bacterial adhesion for periimplantitis. Biomed Res Int 2017: 4732302.

46. Rastenienė R, Pūrienė A, Aleksejūnienè J, Pečiulienė V, Zaleckas L (2015) Odontogenic maxillofacial infections: A Ten-year retrospective analysis. Surg Infect (Larchmt) 16: 305-312. [Crossref]

47. Giannelli M, Landini G, Materassi F, Chellini F, Antonelli A, et al. (2016) The effects of diode laser on Staphylococcus aureus biofilm and Escherichia coli lipopolysaccharide adherent to titanium oxide surface of dental implants. An in vitro study. Lasers Med Sci 31: 1613-1619. [Crossref]

48. Chen CJ, Ding SJ, Chen CC (2016) Effects of surface conditions of titanium denta implants on bacterial adhesion. Photomed Laser Surg 34: 379-388. [Crossref]

49. Tanasiewicz M, Twardawa H (2011) The importance of antibacterial agents in caries prophylaxis. Vol. I. Selected antibacterial media carriers. TwójPrzegl Stomatol 6: 48-53.

50. Marsh PD (2012) Contemporary perspective on plaque control. Br Dent J 212: 601606. [Crossref]

51. Ge L, Li Q, Wang M, Ouyang J, Li X, et al. (2014) Nanosilver par-ticles in medica applications: synthesis, performance, and toxicity. Int J Nanomedicine 9: 2399-2407. [Crossref]

52. Yamamoto K, Ohashi S, Aono M, Kokubo T, Yamada I, et al. (1996) Antibacteria activity of silver ions implanted in $\mathrm{SiO} 2$ filler on oral streptococci. Dent Mater 12: 227-229. [Crossref]

53. Yoshida K, Tanagawa M, Matsumoto S, Yamada T, Atsuta M (1999) Antibacterial activity of resin composites with silver-containing materials. Eur J Oral Sci 107: 290296. [Crossref]

54. Zhang K, Cheng L, Imazato S, Antonucci JM, Lin NJ, et al. (2013) Effects of dual antibacterial agents MDPB and nano-silver in primer on microcosm biofilm, cytotoxicity and dentine bond properties. J Dent 41: 464-474. [Crossref]

55. Strużycka I (2014) The oral microbiome in dental caries. Pol J Microbiol 63: 127-135. [Crossref]

56. Kasraei S, Sami L, Hendi S, Alikhani MY, Rezaei-Soufi L, et al. (2014) Antibacterial properties of composite resins incorpora-ting silver and zinc oxide nanoparticles on Streptococcus mutans and Lactobacillus. Restor Dent Endod 39: 109-114.

57. Bahador A, Pourakbari B, Bolhari B, Hashemi FB (2015) In vitro evaluation of the antimicrobial activity of nanosilver-mineral trioxide aggregate against frequent anaerobic oral pathogens by a membrane-enclosed immersion test. Biomed J 38: 77-83. [Crossref]

58. Ledzion S, Pawlicka H (2005) Assessment of the tightness of root canal fillings with GuttaFlow ${ }^{\circledR}$. CzasStomat 58: 551-554.

59. Kolenbrander PE, Andenser RN, Blehert DS, Egland PG, Foster JS, et al. (2002) Communication among oral bacteria. Microbiol Mol Biol Rev 66: 486-505. [Crossref] 
60. Marsh PD, Bradshaw DJ (1995) Dental plaque as a biofilm. J Ind Microbiol 15: 169175.

61. Strużycka I, Stępień I (2009) Biofilm - a new understanding of the microbiology NowaStomatol 9: 85-89.

62. He XS, Shi WY (2009) Oral microbiology: past, present and future. Int J Oral Sci 1: 47-58. [Crossref]

63. Smith GL, Socransky SS, Smith CM (1989) Rapid method for the purification of DNA from subgingival microorganisms. Oral Microbiol Immunol 4: 47-51. [Crossref]

64. Oda Y, Hayashi F, Wakita A, Nagatani Y, Okada M (2017) Five-year longitudinal study of dental caries risk associated with Streptococcus mutans and Streptococcus sobrinus in individuals with intellectual disabilities. J Oral Sci59: 39-46. [Crossref]

65. Schupper AJ, Jiang W, Coulter MJ, Brigger M, Nation J (2018) Intracranial complications of pediatric sinusitis: Identifying risk factors associated with prolonged clinical course. Int J PediatrOtorhinolaryngol 112: 10-15. [Crossref]

66. Subramani P, Raja V, Lingaiah BB, Madappa BP, Chakravarthy H (2014) Group G Streptococci in association with brain abscess: a rare occurrence. J Infect Dev Ctries 8: $1488-1490$

67. Szyfter W, Bartochowska A, Borucki Ł, Maciejewski A, Kruk-Zagajewska A (2018) Simultaneous treatment of intracranial complications of paranasal sinusitis. Eur Arch Otorhinolaryngol 275: 1165-1173. [Crossref]

68. Mazur E, Czerwińska E, Korona-Głowniak I, Grochowalska A, Kozioł-Montewka M (2015) Epidemiology, clinical history and microbiology of peritonsillar abscess. Eur J Clin Microbiol Infect Dis 34: 549-554. [Crossref]
69. Megalamani SB, Suria G, Manickam U, Balasubramanian D, Jothimahalingam S (2008) Changing trends in bacteriology of peritonsillar abscess. J Laryngol Otol 122: 928-930. [Crossref]

70. Hong P, Liu CM, Nordstrom L, Lalwani AK (2014) The Role of the Human Microbiome in Otolaryngology-Head and Neck Surgery: A Contemporary Review. Laryngoscope 124: 1352-1357. [Crossref]

71. Mortimore S, Wormald PJ, Oliver S (1998) Antibiotic choice in acute and complicated si-nusitis. J Laryngol Otol 112: 264-268. [Crossref]

72. Rudloe TF, Harper MB, Prabhu SP, Rahbar R, Vanderveen D, et al. (2010) Acute periorbital infections: Who needs emergent imaging? Pediatrics 125: e719-e726. [Crossref]

73. Flam JO, Platt MP, Sobel R, Devaiah AK, Brook CD, et al. (2016) Association of oral flora with orbital complications. Am J Rhinol Allergy 30: 257-260. [Crossref]

74. Eykyn SJ (1997) Infective endocarditis: some popular tenets debunked? Heart 77: 191193. [Crossref]

75. Allegrini D, Reposi S, Nocerino E, Pece A (2017) Odontogenic orbital cellulitis associated with cavernous sinus thrombosis and pulmonary embolism: a case report. $J$ Med Case Rep 11: 164. [Crossref]

76. Li E, Distefano A, Sohrab M (2018) Necrotizing orbital cellulitis secondary to odontogenic Streptococcus constellatus. Ophthalmic Plast Reconstr Surg 34: e160-e162. [Crossref]

Copyright: (C2020 Michalik M. This is an open-access article distributed under the terms of the Creative Commons Attribution License, which permits unrestricted use, distribution, and reproduction in any medium, provided the original author and source are credited. 